\title{
IV. Paul Reynaud im Ersten Weltkrieg
}

Daß die kriegerische „Urkatastrophe“ des 20. Jahrhunderts, die soldatische Erfahrung von permanenter unmittelbarer Existenzgefährdung und Tod, der Verlust naher Angehöriger und das Erlebnis der Verwüstung heimatlicher Landstriche Paul Reynaud ebenso tief prägte wie eine ganze Generation französischer Frontsoldaten, zeichnet sich in den wenigen erhaltenen Äußerungen ab, vor allem in den Resten seines Frontbriefwechsels. ${ }^{1}$ Anders aber als dem Großteil jener "génération du feu", und anders auch als einem Teil der politischen Klasse, mit der Reynaud nach 1918 in engen Kontakt treten sollte, blieben ihm das "Grabenerlebnis“ und der längere Dienst in einer Kampfeinheit erspart. An den persönlichen Solidaritäten und den Ansätzen zu einer gemeinsamen Mentalität der Frontgeneration, die daraus erwuchsen und die in der Zwischenkriegszeit bedeutende historische Wirkmächtigkeit erlangten, hatte Reynaud daher nur in begrenztem Maße Anteil. Noch im Jahre 1940 etwa konnte er dem Image des ,ancien combattant“, das ein Edouard Daladier geschickt nutzte, um Autorität und Kompetenz in militärischen Fragen zu demonstrieren, wie auch dem daraus resultierenden Rückhalt seines politischen Konkurrenten in Frontkämpferkreisen und in der Generation der neuerlich zu den Waffen Gerufenen nur wenig entgegensetzen. ${ }^{2}$

Reynauds Kriegserleben unterschied sich von dem der breiten Masse seiner Generation, da er aufgrund seiner ausgesprochen privilegierten Verwendung den Kontakt mit der Sphäre des "Zivilisierten“, mit geistiger Tätigkeit und auch mit dem Bereich des Politischen nicht lange verlor. So sind in den erhaltenen Aufzeichnungen, die sein Kriegserlebnis widerspiegeln, persönliche Betroffenheit und Fronterlebnis weitaus weniger faßbar als die Lust am Erkennen und Bewerten politischer Zusammenhänge, Vermittlerleidenschaft und nicht zuletzt ein Zug von Weltläufigkeit und Abenteurertum.

Als Sergent und Mitglied der Territorialarmee leistete er zunächst Dienst in verschiedenen motorisierten Nachschub- und Krankentransporteinheiten, bevor er nach schwerer Krankheit ab Juli 1917 für einige Monate durch das Kriegsministerium abgestellt wurde, um als Mitarbeiter eines direkt dem Ministerpräsidenten

1 Einige Überreste seines Briefwechsels mit einem Familienmitglied, vermutlich seinem Cousin Maurice Gassier, von Juni bis Dezember 1916 finden sich im Nachlaß (AN, 74 AP 6).

2 Der sechs Jahre jüngere Edouard Daladier, im Juli 1914 Bürgermeister von Carpentras (Vaucluse), beendete den Krieg als mehrfach verwundeter und ausgezeichneter Frontsoldat (Elisabeth du Réau, Edouard Daladier. 1884-1970, Paris 1993, S. 28-36); zu Geschichte, sozialer Struktur und Mentalität der franz.ösischen Frontkämpfervereinigungen vgl. Antoine Prost, Les anciens combattants et la société française (1914-1939), 3 Bände, Paris 1977.

Bezeichnend für das schwierige Verhältnis Reynauds zu den Frontkämpfern ist der Tagebucheintrag Jean-Paul Sartres vom 28. März 1940: „Les soldats, ici, reprochent à Reynaud de n'avoir pas dit un mot dans son allocution radiodiffusée sur , l'héroïsme de nos vaillants soldats'. ,Daladier n'y manquerait jamais, lui!" disent-ils avec regret." (Jean-Paul Sartre, Les carnets de la drôle de guerre. Novembre 1939 - Mars 1940, Paris 1983, S. 431). 
unterstellten Komitees an der Rekrutierung slawischer Freiwilliger mitzuwirken. ${ }^{3}$ Die Bewährung in dieser Funktion verschaffte ihm im Sommer 1918 die Chance, in Begleitung des französischen Oberkommandierenden der neugegründeten tschechischen Armee seine Fähigkeiten zu erproben.

Mit Ausbruch des Ersten Weltkriegs war die seit mehr als einem halben Jahrhundert schwebende Frage einer tschechischen Nationalstaatsgründung neu in Bewegung geraten. ${ }^{4}$ Vor dem Hintergrund der kriegsbedingten Einschränkung parlamentarischer und staatsbürgerlicher Rechte in Österreich-Ungarn und konfrontiert mit der Gefahr der Rücknahme bereits erreichter Reformen im deutschtschechischen Verhältnis, zweifelte man in den tschechischen Bevölkerungsteilen der Habsburgermonarchie zunehmend an der Ausgleichsbereitschaft der Staatsführung. Neben einer wachsenden innerösterreichischen Oppositionsbewegung in Böhmen und Mähren entstand bereits seit den ersten Kriegsjahren eine exiltschechische Bewegung, die sich auf Kolonien in den USA, in Großbritannien, Frankreich und Rußland stützen konnte und durch die unermüdliche Reise- und Vermittlungstätigkeit dreier Exilpolitiker - Thomas Masaryk, Edvard Beneš und Milan R. Stefánik - bis Sommer 1917 weitgehend auf das nationaltschechische Programm Masaryks und Beneš' eingeschworen wurde. So hatte man bereits im Februar 1916 in Paris, das die stärkste tschechische Kolonie in Westeuropa beherbergte, einen "Conseil national des pays tchèques“ gegründet, dem künftig die einheitliche Interessenvertretung gegenüber der Entente zukommen sollte. Man entfaltete - zunächst in Paris, dann nach ähnlichem Muster auch in London - eine intensive Propagandatätigkeit, die Kontakte zu slawophilen Kreisen in Presse, Universität und Politik schuf und nutzte, um die eigenen, der Öffentlichkeit noch weitgehend unbekannten Anliegen bekanntzumachen. ${ }^{5}$

$\mathrm{Zu}$ definitiven Zusagen war die französische Regierung, der Hauptansprechpartner des Pariser Nationalrats, indes gegenüber der exiltschechischen Bewegung so lange nicht bereit, als die Möglichkeit eines Sonderfriedens mit Österreich-Ungarn und damit eine möglicherweise kriegsentscheidende Spaltung der Mittelmächte nicht auszuschließen war. ${ }^{6}$ Angesichts dieser Konstellation setzten Masaryk und Beneš zur Durchsetzung ihrer politischen Ziele zunehmend auf den Aufbau einer tschechischen Armee und deren Eingreifen in den Krieg gegen die Mittelmächte. In Verhandlungen, die Edvard Beneš zwischen Juni und September 1917 mit dem Quai d'Orsay führte, einigte man sich darauf, tschechische Truppen

3 „Etat des Services de M. Reynaud, Jean, Paul“ (SHAT, Dossier Reynaud, Paul, No. 42325); vgl. auch Reynaud, Mémoires I, S. $109 \mathrm{f}$.

+ Die folgenden Ausführungen beruhen im wesentlichen auf der nach wie vor besten Überblicksdarstellung: Ernst Birke, Der Erste Weltkrieg und die Gründung der Tschechoslovakei 1914-1919, in: Karl Bosl (Hg.), Handbuch der Geschichte der böhmischen Länder. Band III, Stuttgart 1968, S. 239-446, hier: 274-446, sowie auf: Piotr S. Wandycz, France and Her Eastern Allies 1919-1925. French-Czechoslovak-Polish Relations from the Paris Peace Conference to Locarno, Minneapolis 1962, S. 3-26.

Vgl. dazu auch: Frantisek Kolár, Edouard Beneš et le Conseil national tchécoslovaque, in: Guerres mondiales et conflits contemporains 169 (1993), S. 9-15; Martin Nechvatal, La naissance d'une armée tchécoslovaque en France, ebenda, S. 37-41; Jörg K. Hoensch, Geschichte der Tschechoslowakischen Republik 1918-1978, Stuttgart u. a. 21978, S. 11-26.

5 Vgl. Birke, Gründung, S. 311; Kolár, Beneš, S. 9-11.

6 Vgl. Birke, Gründung, S. 316 und 359f.; Wandycz, Eastern Allies, S. 9-11. 
aus aller Welt in Frankreich zusammenzuziehen, als unabhängige Einheiten im Rahmen der französischen Armee der alleinigen politischen Führung durch den Pariser Nationalrat zu unterstellen und an der Westfront einzusetzen. ${ }^{7}$ Dabei galt das besondere Augenmerk sowohl der exiltschechischen Führung als auch der französischen Organisatoren des ambitiösen Planes zunächst dem russischen Kriegsschauplatz, baute man doch auf die Remobilisierung des enormen, in russischen Kriegsgefangenenlagern festgesetzten Potentials an tschechischen, ehemals unter österreichischer Fahne kämpfenden Soldaten. Nach Rekrutierungsreisen Stefániks und Masaryks im Jahre 1916 und im Sommer 1917 umfaßte das mit Genehmigung des russischen Generalstabs aufgestellte Kontingent bis Ende 1917 etwa acht Regimenter in zwei Divisionen, von denen im Frühjahr 1918 Teile in Kämpfe mit den in die Ukraine vorrückenden deutschen Truppen verwickelt wurden. ${ }^{8}$ Zum Oberbefehlshaber der tschechischen Truppen wurde General Maurice Janin, ehemals Leiter der französischen Militärmission in Rußland, ernannt, der im August 1918 nach Sibirien abreiste. ${ }^{9}$

Mit dem Ausbruch der russischen Revolution und dem Friedensschluß von Brest-Litowsk im März 1918 hatten sich die diplomatischen Rahmenbedingungen wie auch die innerrussischen Gegebenheiten, die für das weitere Schicksal der tschechischen Legion bestimmend sein sollten, grundlegend geändert. Seitens der Allierten begann nun im Kontext der Verhandlungen um die Wiedererrichtung einer Front im Osten ein intensives Tauziehen um die weitere Verwendung dieser Truppen, die nach dem Ausscheiden des russischen Bündnispartners den einzigen nennenswerten alliierten Verband auf russischem Boden bildeten. Dabei gab die französische Führung, ursprünglich angesichts der bedrohlichen Lage an der Westfront im Frühjahr 1918 an der Einschiffung einer möglichst großen Zahl tschechischer Soldaten nach Frankreich interessiert, bis zum Sommer 1918 ihren zähen Widerstand gegenüber der britischen Forderung, die Legion als Kern einer alliierten Interventionstruppe in Sibirien zu belassen, weitgehend auf. ${ }^{10}$ Für diese

7 Über die langwierigen Verhandlungen berichtet Beně̌ ausführlich in seinen Memoiren: Edvard Beneš, Der Aufstand der Nationen. Der Weltkrieg und die tschechoslowakische Revolution, Prag 1928, S. 214-231, 351-353. Vgl. auch Birke, Gründung, S. 361.

Am 21. April 1918 schloß Stefánik mit der italienischen Regierung ein ähnliches Abkommen über die Bildung einer unabhängigen tschechoslowakischen Armee aus ehemaligen Kriegsgefangenen ab (Unterberger, United States, S. 63).

8 Vgl. Birke, Gründung, S. 314 und $351 \mathrm{f}$.

9 Maurice Janin, im Dezember 1915 zum Brigadegeneral ernannt, war nach Kriegseinsätzen an der Westfront und einer Verwendung im Stab des Generals Joffre im April 1916 als ausgewiesener Rußlandexperte zum Leiter der französischen Militärmission in Petrograd ernannt worden. Bis zu seiner Abreise Ende 1917 leistete er vor allem Vermittlerdienste zwischen dem französischen und dem russischen Oberkommando und versuchte, den Kriegseintritt Rumäniens an der Seite der Entente zu erreichen. Im August 1916 machte Janin hier die Bekanntschaft von Milan R. Stefánik, woraus sich eine langjährige enge Zusammenarbeit entwickeln sollte (vgl. Dusan Kovác, Deux hommes dans la Grande Guerre: Maurice Janin et Milan Rastislav Stefánik, in: Guerres mondiales et conflits contemporains 169 (1993), S. 51-58).

$10 \mathrm{Vgl}$. zu den langwierigen französisch-englischen Verhandlungen um die Verwendung der tschechischen Legion ab Februar 1918: Michael Jabara Carley, Revolution and intervention: the French Government and the Russian civil war, 1917-1919, Kingston 1983, S. $33 \mathrm{ff}$.

Die Studie von Michael Kettle, Russia and the Allies 1917-1920. Band 2: The Road to Intervention. March-November 1918, London/New York 1988, datiert die Wende der französischen Haltung anhand englischen Archivmaterials auf Anfang August (Kettle, Russia, S. 49-302, hier: 302). Unter Zuziehung einzelner französischer Archivalien gibt die Gespräche wieder: Betty Miller Unterber- 
Entscheidung waren der Entschluß Wilsons, amerikanische Truppen in begrenztem Maße an der Intervention zu beteiligen, ebenso maßgeblich wie die im Sommer 1918 sich stabilisierenden Transporte amerikanischer Truppen nach Europa und das endgültige Scheitern der deutschen Frühjahrsoffensive an der Westfront im Juli. In einer Direktive an General Janin vom 7. August 1918 wies Clemenceau die tschechischen Verbände zu enger Zusammenarbeit mit den alliierten Landungstruppen an, um so, ausgehend von der sibirischen Transkontinentalen, ein dichtes Widerstandsnetz gegen die deutsche Ausdehnung nach Osten zu knüpfen. ${ }^{11}$

Reynauds Tätigkeit in der Rekrutierungskommission hatte ihm nicht nur die Bekanntschaft mit Edvard Beneš eingetragen, sondern auch die besondere Aufmerksamkeit seines militärischen Vorgesetzten, General Maurice Janin. Intelligenz, Takt, Diskretion und nicht zuletzt Welterfahrenheit waren es, die dieser offenbar an seinem Mitarbeiter schätzte, dessen er sich im August 1918 erinnerte, als es darum ging, einige Offiziere zur Einrichtung eines regelmäßigen Kurierdienstes zwischen Paris und der sibirischen Mission auszuwählen. Nach fünfmonatiger weiterer Verwendung als Zugführer in einer Sanitätstransporteinheit, die während der deutschen Frühjahrsoffensive an der Aisne 1918 schwere Verluste erlitt, wurde Reynaud nach Paris zurückbeordert und auf ausdrückliches Verlangen Janins dessen Delegation mit Wirkung vom 31. August 1918 zugeteilt. ${ }^{12}$

Am 10. September 1918 verließ Reynaud Frankreich von Brest aus an Bord des Passagierschiffs "France“, das als Truppentransporter amerikanische Soldaten nach Europa gebracht hatte. Mit ihm traten nur einige als Instruktoren für die amerikanische Armee bestimmte Offiziere die Reise auf dem menschenleeren Schiff an - begleitet von ständiger Furcht vor der Torpedierung durch deutsche Unterseeboote und, was Reynaud anging, in wehmütiger Erinnerung an den Beginn seiner Weltreise elf Jahre zuvor. ${ }^{13}$ Nach Zwischenaufenthalten in New York

ger, The United States, Revolutionary Russia, and the Rise of Czechoslovakia, Chapel Hill/London 1989, passim. Sie sieht einen Wendepunkt der französischen Außenpolitik im Hinblick auf eine Verwendung der tschechischen Truppen in Sibirien bereits Ende Juni 1918 erreicht (Unterberger, United States, S. 212f. und 231-235). Vgl. auch Dies., Woodrow Wilson and the Russian Revolution, in: Arthur B. Link (Hg.), Woodrow Wilson and a Revolutionary World, 1913-1921, Chapel Hill 1982, S. 49-104. Beide Autoren analysieren den diplomatischen Prozeß, ohne umfangreicheres französisches Material zu benutzen.

11 „Instructions pour M. le Général Janin“, 7. 8. 1918, S. 1f. (SHAT, 7 N 628). Kettle, Intervention, S. $302 \mathrm{f}$ datiert die Anweisungen auf den 5.8. und gibt sie anhand einer Kopie wieder, die Clemenceau am 19. August an Lloyd George übersandte.

12 Reynaud kommandierte die 128. Section Sanitaire vom 1. April bis 30. August 1918 („Etat des Services de M. Reynaud, Jean, Paul“; SHAT, Dossier Reynaud, No. 42325);

In einem Schreiben an den Generalstab hatte Janin im August sechs Offiziere als Verbindungsleute angefordert. Zwei davon, darunter Reynaud, sollten die Mission sofort begleiten. In dem Schreiben hieß es unter anderem: „Les officiers courriers n'étant pas uniquement convoyeurs de colis mais apportant avec eux une série de nouvelles, d'atmosphère de la Patrie, emportant également une impression de ce qu'ils ont vu, je serais désireux de voir figurer à cet emploi quelques officiers dont j'ai pu apprécier déjà l'intelligence, le tact, la discrétion nécessaires et qui sont habitués aux voyages en pays étrangers." (Janin an Etat-Major de l'Armée, Bureau Slave, 23. 8. 1918; SHAT, 7 N 628).

In einem Telegramm an den kommandierenden General der Nord- und Nordostarmeen hatte Janin zuvor bereits persönlich um die Freistellung Reynauds gebeten (Janin an Général Comdt. en Chef, No. 1784, 14. 8. 1918; ebenda). Vgl. dazu auch Reynaud, Mémoires I, S. 109 f.

13 Vgl. den Eintrag Reynauds in sein Reisetagebuch vom 10. September 1918 (AN, 74 AP 6). Zwi- 
und Washington, die er zu Kontakten mit der dortigen französischen Kolonie nutzte, nach der Reise durch den amerikanischen Kontinent und der Überquerung des Pazifiks stieß Reynaud Ende Oktober in Tokio zu Janin, in dessen Begleitung er am 13. November 1918 in Vladivostok eintraf. ${ }^{14}$ Im Gefolge von General Stefánik, der sich als neuernannter Verteidigungsminister der mittlerweile gegründeten tschechoslowakischen Republik der Mission Janin angeschlossen hatte, brach er fünf Tage später ins Landesinnere auf.

Dort hatten sich seit April die Rahmenbedingungen für einen Abtransport der tschechischen Legion nach Europa grundlegend verändert, nachdem die sowjetrussischen Behörden den Militärzügen durch Repressalien die Weiterfahrt erschwert hatten. Einzelne blutige Zwischenfälle waren bis Ende Mai in eine allgemeine Aufstandsbewegung der tschechischen Truppen gegen örtliche Sowjets gemündet, in deren Verlauf die Legion bis Ende August die gesamte transsibirische Bahnlinie von der Wolga bis nach Vladivostok unter ihre Kontrolle gebracht hatte. Zudem war sie in Kämpfe an der Seite „weißer“ Truppen verwickelt worden, die seit November 1917 gegen die Bolschewiki vorgingen..$^{15}$ Damit hatte sie sich nicht nur von Masaryks Vorgabe, in den innerrussischen Auseinandersetzungen neutral zu bleiben, gelöst; die Truppe, die während einiger Monate von der Außenwelt und von alliierter Führung abgeschnitten geblieben war, hatte sich darüber hinaus politisch gespalten und Selbstverwaltungsorgane eingerichtet, die den Anspruch der Moskauer Außenstelle des Pariser Nationalrats auf politische Führung des Korps bewußt beschnitten hatten. ${ }^{16}$ Mit den ersten Niederlagen gegen die bolschewistischen Truppen ab Anfang September begann die Moral in der Legion mehr und mehr zu schwinden. Nachdem man von der Gründung der tschechischen Republik am 28. Oktober 1918 erfahren hatte, griff Kriegsmüdigkeit und der Wunsch um sich, möglichst bald den Heimweg anzutreten. ${ }^{17}$

Während der knapp dreiwöchigen Bahnreise nach Tscheljabinsk, dem Hauptquartier der tschechischen Legion und vorläufigen Ziel Stefániks, entwickelte sich zwischen dem 38jährigen General und dem um zwei Jahre älteren Leutnant allmählich ein Vertrauensverhältnis, das sich in mehreren längeren Gesprächen manifestierte, über die Reynaud ausführliche Mitschriften anfertigte. Offiziell lediglich als Kurier vorgesehen, erschloß er sich in Abwesenheit des in Vladivostok festgehaltenen Janin nach und nach einen weiteren Aufgabenbereich. Nicht zuletzt aufgrund seiner noch in Paris erworbenen Sachkenntnis wuchs er in die Rolle eines Vermittlers zwischen französischen und tschechischen sowie tschechischen und polnischen Belangen, der die Parteien mit diplomatischem Geschick im Sinne französischer Interessen auszusöhnen und miteinander zu koordinieren bemüht war. So versuchte er, den schwankenden und auf seine Popularität bei den heim-

schen dem 10. September 1918 und dem 14. Februar 1919 führte er ein Journal, dem er persönliche Eindrücke, Gesprächsnotizen und Kopien offizieller Dokumente anvertraute, die im Laufe seiner Tätigkeit entstanden waren. Siehe auch Reynaud, Mémoires I, S. $110 \mathrm{f}$.

$14 \mathrm{Vgl}$. die Tagebucheinträge zwischen 18. September und 13. November 1918 (AN, 74 AP 6).

15 Vgl. hierzu Thunig-Nittner, Tschechoslowakische Legion, S. 45-80; Birke, Gründung, S. 356-358; Bradley, Czechoslovak Legion, S. 77-105.

16 Vgl. Thunig-Nittner, Tschechoslowakische Legion, S. 52-55 und 67-69; Bradley, Czechoslovak Legion, S. 94.

17 Vgl. Thunig-Nittner, Tschechoslowakische Legion, S. 76-80. 
kehrwilligen Truppen bedachten Stefánik von der Notwendigkeit des Verbleibs in Sibirien zu überzeugen ${ }^{18}$, seine Beschwerden über nicht eingehaltene alliierte Versprechen zu entkräften und tschechische Ressentiments gegen die Bildung polnischer Verbände in Sibirien abzubauen. ${ }^{19}$ Zwar blieb der Erfolg seiner Bemühungen begrenzt: nach einer vierwöchigen Inspektionsreise veranlaßte Stefánik noch im Januar 1919 den Rückzug der erschöpften Legion aus den Kämpfen.20 Auch wurde dem widerstrebenden Reynaud bereits am 7. Dezember mitgeteilt, daß er auf Verlangen seines Schwiegervaters als Kurier nach Frankreich zurückzukehren habe. ${ }^{21}$ In der Folge mit verschiedenen vertraulichen militärisch-diplomatischen Missionen betraut, die er auf seinem Rückweg nach Vladivostok wahrnahm²2, agierte Reynaud indes weiter so geschickt und engagiert, daß ihm zumindest die persönliche Genugtuung zuteil wurde, durch Janin für die Aufnahme in die Ehrenlegion vorgeschlagen $\mathrm{zu}$ werden..$^{23}$

Nach einer nicht ohne abenteuerliche Zwischenfälle verlaufenen Rückreise mit der Transsibirischen Eisenbahn von Omsk nach Vladivostok und per Schiff über Hiroshima und Tokio nach Washington hatte Reynaud ein weiteres Mal Gelegenheit, diplomatische Fähigkeiten unter Beweis zu stellen. Bereits im Oktober 1918 hatte Janin den Quai d'Orsay für seinen Vorschlag gewinnen können, dem Informationsdefizit in den USA im Hinblick auf die Vorgänge in Sibirien und Rußland gezielt entgegenzuwirken. Jene "mission de propagande" setzte Reynaud Anfang 1919 mit Energie und Einfallsreichtum um. ${ }^{24}$ In Begegnungen mit Vertretern der französischen Hohen Kommission, mit Botschafter de Chambrun, Offizieren des amerikanischen Geheimdienstes und des Generalstabs gab er zwischen dem 10. und 12. Februar 1919 aktuelle Lageberichte über die Situation in Sibirien, die im wesentlichen den Beobachtungen und Analysen entsprachen, die er in seinem Abschlußbericht an Clemenceau vom 15. Februar noch in New York niederlegte. ${ }^{25}$

Der kenntnisreich, doch eigenwillig verfaßte Bericht Reynauds reicht, ohne die mündliche Lageeinschätzung zu vernachlässigen, die er durch Janin zur Berichterstattung in Paris mit auf den Weg gegeben bekam, in seinen konstruktiven Überlegungen und Schlußfolgerungen weit über jene hinaus. ${ }^{26}$ Ausgehend von

18 Vgl. die Tagebucheintragung Reynauds vom 21. November 1918 sowie seine Notizen über ein ausführliches Gespräch mit Stefánik am 22. November 1918 (AN, 74 AP 6).

$19 \mathrm{Vgl}$. Reynauds Unterredungen mit Stefánik vom 27. November sowie seinen Rechenschaftsbericht gegenüber dem General am 1. und 2. Dezember 1918 (Ebenda).

20 Thunig-Nittner, Tschechoslowakische Legion, S. $81 \mathrm{f}$.

21 Siehe dazu den Tagebucheintrag vom 7. Dezember 1918 (AN, 74 AP 6). In seinen Memoiren stellt Reynaud seine Abreise als Akt eigenen Willens dar (Reynaud, Mémoires I, S. 123).

22 Vgl. die Anweisung Janins an Reynaud vom 17. Dezember 1918 und dessen Berichte vom 30. Dezember 1918 und 2. Januar 1919 (AN, 74 AP 6).

23 Vgl. Reynauds Notizen vom 20. und 21. Dezember 1918 (AN, 74 AP 6). Die Ernennung zum Chevalier de la Légion d'Honneur erfolgte erst per Dekret vom 5.11. 1928, da man seine Verdienste während des Einsatzes in Frankreich nicht als ausreichend erachtete, bzw. den fehlenden Fronteinsatz bemängelte (Etat-Major de l'Armée. 2e Bureau. Mémoire de proposition pour Chevalier de la Légion d'Honneur, Juni 1919 bzw. Januar 1920; SHAT, Dossier Reynaud, No. 42325).

${ }^{24}$ MAE an Etat-Major de l'Armée - Bureau Slave, No. 3703 vom 1. 10. 1918 (SHAT, 7 N 628).

$25 \mathrm{Vgl}$. zu seinem Aufenthalt in Washington und seinen Unterredungen mit General Collardet, Oberst Langlois und Botschaftsrat de Chambrun die Tagebucheintragungen vom 10., 11. und 12. Februar 1919 (AN, 74 AP 6).

26 Die Instruktionen, die Janin Reynaud mit auf den Weg gab, betrafen vor allem die eingeschränkte Kampftauglichkeit der tschechischen Legion und der weißrussischen Truppen und zeichneten ein 
einer vernichtenden Analyse des sibirischen und russischen „Volkscharakters“, dem Wertebindung oder Patriotismus fremd seien, kritisiert Reynaud hier die bürgerliche Oberschicht des Landes. Sie ermangle jeder Befähigung zur politischen Führung und scheue wie die breite Masse des Volks insbesondere im östlichen Landesteil um ihrer wirtschaftlichen Interessen willen selbst ein Arrangement mit den Bolschewisten nicht: „Quoique le Sibérien soit un peu plus avancé que le Russe, les facteurs généraux leurs sont communs. Ils dérivent d'une amoralité complète aux points de vue familial, commercial et politique. L'absence totale de patriotisme qui en dérive est connue [...]". ${ }^{27}$ Angesichts der Schwäche der tschechischen Legion, der mangelnden Verläßlichkeit ihrer weißrussischen Verbündeten und der geringen französischen Präsenz sei ein Eingreifen alliierter Truppen in Divisionsstärke unabdingbar, um das weitgehend antibolschewistisch eingestellte West- und Zentralsibirien durch einen „cordon sanitaire“ zu schützen und das Vordringen der bolschewistischen Propaganda nach Westen zu verhindern. ${ }^{28}$ Kernstück dieser Strategie müsse der Verzicht der USA auf ihr bloß halbherziges Engagement in Rußland sein. Das Vordringen amerikanischer Wirtschaftskraft auf den sibirischen Markt könne gleichermaßen den bisherigen Konkurrenten Deutschland verdrängen und bolschewistischer Agitation den Boden entziehen: „[...] ce serait un premier pas dans la reconstitution de la Russie avec la seule base qui puisse être proposée à un peuple dépourvu de patriotisme: l'intérêt social. C'est seulement en enrichissant les Sibériens qu'on peut les rééduquer à la civilisation. "29 Falls diese Aktion nicht zustande komme, müsse dagegen mit dem Zusammenbruch der Front, mit der Ausweitung des bolschewistischen Einflußbereichs nach Westen und schließlich mit der propagandistischen Intervention der Bolschewiki in der moslemischen Welt und im französischen Einflußbereich Afrikas gerechnet werden..$^{30}$

Vor dem Hintergrund der interventionistischen Grundhaltung seiner Regierung entwickelte Reynaud hier also eine Argumentationsstrategie, die zwei wesentliche Elemente umfaßte. Zum einen vertrat er gegenüber Paris den Standpunkt Janins, der mehrfach vergeblich bei Clemenceau um Entsendung von Truppen zur Unterstützung der sibirischen Front ersucht hatte. Der Ministerpräsident hatte sich im Oktober 1918 zwar entschieden, die französische Osteuropapolitik gleichermaßen auf ein Zurückdrängen des Bolschewismus wie die Bekämpfung der Mittelmächte auszurichten. Angesichts des geschwächten Kriegspotentials Frankreichs war er jedoch nur zu begrenzter Intervention mit eigenen Kontingenten bereit und dies nur im südrussischen Bereich, wo er die englische Konkurrenz um Einflußsphären im Kaukasus und im Nahen Osten fürchtete. Im übrigen

eher pessimistisches Bild: „[...] Dites à Paris que situat[ion] dangereuse. En face, les Bolch[éviques] consolidés, plus nombreux, mieux commandés. “

Reynaud kommentierte die ihm anvertrauten Mitteilungen lakonisch: „Il ne me dit rien de vraiment sérieux“ (Tagebucheinträge Reynauds vom 21. Dezember 1918; ebenda).

27 „Le Lieutenant Reynaud de l'E.M.A. 2e bureau C.E. à Monsieur le Président du Conseil, Ministre de la Guerre", Februar 1919, S. 6 (AN, 74 AP 6). In Auszügen findet sich der Bericht auch wiedergegeben in: Reynaud, Mémoires I, S. 126-128.

29 Ebenda, S. 18 f.

30 Ebenda, S. 5. 
setzte Clemenceau auf diplomatisches Vorgehen, um insbesondere die USA von der Notwendigkeit eines interalliierten Eingreifens zu überzeugen. ${ }^{31}$ In diesem Konzept, das in der Ukraine und in Westsibirien lediglich defensiv ausgerichtete Hilfe zur Selbsthilfe für die weißrussischen Verbände vorsah, hatten die Beistandsersuchen Janins und anderer keinen Platz. ${ }^{32}$

Deshalb zielte Reynauds Vorgehensvorschlag zum zweiten darauf, Clemenceaus starre Option für ein bewaffnetes Vorgehen gegen die bolschewistische Bewegung durch ein an deren sozio-ökonomischen Ursachen orientiertes, effektiveres Eindämmungsmodell zu ergänzen. Es konnte angenommen werden, daß ein solches, auf handelspolitische Interessen gestütztes, differenziertes Vorgehen dazu beitragen würde, den interventionsfeindlichen Teilen der amerikanischen Führung und Öffentlichkeit ein Eingreifen im revolutionären Rußland in französischem Sinne nahezubringen.

Tatsächlich hatte sich der amerikanische Präsident, aufgrund prinzipieller Bedenken ein dezidierter Gegner jeder umfassenden alliierten Intervention, im Juli 1918 nur unter großen Vorbehalten zur Entsendung eines auf 7000 Mann begrenzten Kontingents bereitgefunden. Dessen Haupteinsatzzweck sollte in der Unterstützung der tschechischen Legion bis zu ihrem erfolgreichen Abtransport nach Europa und in der Kontrolle des gleichzeitig entsandten japanischen Kontingents liegen. Weder die Wiederherstellung einer Ostfront gegen das deutsche Vorrücken noch ein Eingreifen gegen die Bolschewiki fand demgegenüber seine Billigung. Wenngleich die amerikanische Intervention bis Herbst 1918 de facto mehr und mehr den Charakter einer aktiven Parteinahme im russischen Bürgerkrieg annahm, verweigerte sich Wilson doch hartnäckig den englischen und französischen Pressionen auf Entsendung weiterer Truppen. ${ }^{33}$ Dies hatte der amerikanische Präsident unter anderem auch gegenüber Janin deutlich gemacht, als dieser ihm auf dem Weg nach Sibirien im September 1918 einen entsprechenden Wunsch Clemenceaus überbracht hatte. ${ }^{34}$

Reynauds Abschlußbericht an seinen Regierungschef berücksichtigte diese Konstellation und versäumte es deshalb nicht, auf Handlungsspielräume und Ansatzmöglichkeiten für eine Revision der amerikanischen Haltung hinzuweisen: Sowohl im Generalstab der USA als auch im Kongreß gebe es durchaus gewichtige Stimmen, die mit der Haltung Wilsons nicht einverstanden seien und auf mehr moralische Unterstützung durch Frankreich hofften. ${ }^{35}$

Noch vor der Rückkehr nach Europa wurde Reynaud mit Billigung seines Vorgesetzten Janin erneut selbst in diesem Sinne tätig. In einem Brief an den Herausgeber der New York Times wiederholte er nun öffentlich seine Werbung um Entsendung einer amerikanischen Division zur Unterstützung der "demokratischen“

31 Zur Haltung der Alliierten gegenüber der russischen Frage im Januar 1919: Arno J. Mayer, Politics and Diplomacy of Peacemaking. Containment and Counterrevolution at Versailles, 1918-1919, London 1968, S. 284-343; zur französischen Position: S. 294-308.

32 Vgl. hierzu die Telegramme Clemenceaus an Janin vom 13.12. und 22. 12. 1918, in denen er seine Defensivstrategie in Sibirien erläuterte (abgedruckt bei Mayer, Peacemaking, S. 300 f.).

33 Zu den Motiven und Entscheidungen des Präsidenten im Hinblick auf eine amerikanische Beteiligung an der alliierten Intervention in Rußland vgl. Unterberger, Wilson, S. 57-88.

34 Unterberger, Wilson, S. 77.

35 „Le Licutenant Reynaud...., S. $20 \mathrm{f}$. 
tschechischen Legion gegen das "autokratische“ bolschewistische Regime.36 Deutlich optimistischer als noch in seiner internen Lageanalyse gab er sich hier überzeugt vom baldigen Fall des neuen Regimes und von der Möglichkeit, die Bevölkerung im demokratischen Sinne „umzuerziehen“" ${ }^{37}$ Sein Hinweis auf die immensen Rohstoffvorräte Sibiriens zielte auf die Anhänger einer aktiven amerikanischen Einflußpolitik im Fernen Osten; die Erwähnung amerikanischer Opendoor-Interessen in Sibirien traf ein genuines Anliegen Wilsons: „The question of yesterday was the question of Europe; the question of tomorrow is the question of the Pacific. If the United States is to be a factor in the control of the Pacific, it is essential that the Unites States have a strong position in Siberia - an open-door policy for a fair commercial competition. With the United States well entrenched in Siberia, the position of all the Allies would be strengthened." 38

Als vielseitig verwendbar hatte sich Reynaud erwiesen, als nützlich und effektiv, auch wenn das mittelfristig angestrebte Ziel, die USA stärker zu binden, in der Folge in erster Linie aufgrund der Macht des Faktischen erreicht wurde. Nicht der klare Meinungswandel Wilsons, sondern die Tatsache, daß die Vereinigten Staaten bereits zu intensiv engagiert waren, die Erfahrung auch, daß eine neutrale Position amerikanischer Truppen vor Ort nicht aufrechtzuerhalten war, verstrickte die amerikanische Politik zusehends mehr in den russischen Bürgerkrieg. Bekanntlich aber blieb die schließlich gemeinsam unternommene Unterstützung Koltschaks durch die Alliierten auf längere Sicht erfolglos.

Die Stimme eines unbekannten französischen Leutnants in halb militärischer, halb politischer Mission war in diesem alliierten Tauziehen um die angemessene Politik gegenüber den russischen Bürgerkriegsparteien sicherlich von geringem Gewicht gewesen. Aufschlußreicher als hinsichtlich des interalliierten Entscheidungsprozesses sind die beschriebenen Vorgänge deshalb zur Einschätzung seiner Person selbst.

Loyal gegenüber seinem militärischen Mentor Janin, orientiert an der Strategie Clemenceaus - sein Vorschlag entsprach in den Grundzügen exakt dessen Plan eines "cordon sanitaire“ zum Schutz von Ukraine, Kaukasus und Westsibirien 39 und unter Aufnahme der Kritik republikanischer Kreise in den USA an Wilson hatte Reynaud die These entschlossener alliierter Intervention vertreten. Er hatte seinen Vorschlag exakt in Lage und Auftrag eingepaßt und dabei den verfügbaren Spielraum seiner privilegierten Verwendung genutzt und ausgeweitet. Er hatte es überdies verstanden, auf sich aufmerksam zu machen und offensichtlich war er dabei ganz in seinem Element gewesen. Von der Mehrheitsmeinung in der Öffentlichkeit und der politischen Führung seines Landes brauchte er sich dabei nicht zu entfernen. Seine Position entsprach der interventionistischen Tendenz in der gemäßigten und rechten Presse Frankreichs ebenso wie der Unterstützung, die Cle-

36 "One American division would be perfectly sufficient to table the place of the Czecho-Slovaks and make the front absolutely safe.“ (Durchschlag eines Briefes Reynauds „To the Editor of The New York Times", undatiert, vermutlich vor dem 18. Februar 1919 entstanden, S. 2; AN, 74 AP 6).

37 "They do not know that there is a form of government and civilization which will benefit them. The Allies must teach them this." (Ebenda, S. 3).

38 Ebenda, S. 4. Dazu Unterberger, Wilson, S. 80 f.

39 Vgl. die Beschreibung seiner Absichten, die Clemenceau in einem Telegramm an Janin vom 13. 12. 1918 gab (abgedruckt bei Mayer, Peacemaking, S. 300). 
menceau in der Kammer der Abgeordneten seitens des Zentrums und der rechten Mitte gefunden hatte. Uneins in der Frage der zu wählenden Mittel, stimmte das bürgerliche Lager doch darin überein, daß das bolschewistische Regime so rasch wie möglich eingedämmt und gestürzt werden müsse - bei möglichst geringem Aufwand. ${ }^{40}$ Vor dem Hintergrund dieses breiten, parteiübergreifenden antibolschewistischen Konsensus in Frankreich, der bis in Teile der sozialistischen Partei reichte, kam Reynauds Aktion somit einem weiteren Ausweis verläßlicher republikanischer Gesinnung gleich. ${ }^{41}$

Zwei Jahre nach Ausbruch der russischen Revolution und lange vor deren endgültiger Konsolidierung hatte er durch das Erlebnis des Bürgerkriegs aus nächster Nähe die Überzeugung gewonnen, daß die neue Bewegung genügend Anziehungskraft und steigende politische Wirkmächtigkeit besaß, um die Grundlagen des Gesellschaftsmodells in Gefahr zu bringen, das er favorisierte. Reynaud ging aus dem Weltkrieg als dezidierter Bolschewismus-Gegner hervor, was ebensowenig erstaunt wie die Tatsache, daß ihn neben den politischen Verfolgungen insbesondere die Maßnahmen zur Enteignung der Bevölkerung empörten. Wie selbstverständlich hatte er deshalb eine französische Rußlandpolitik mitvollzogen, die ihren antibolschewistischen Impetus nach dem Waffenstillstand vom November 1918 nicht mehr im Krieg gegen die Mittelmächte verbergen konnte. Die Doktrin vom „Kreuzzug“ gegen das Vordringen der kommunistischen Doktrin nach Westen und zur Rettung universaler zivilisatorischer Werte fand in Reynaud einen willigen und überzeugten Verfechter: „Ce n'est pas seulement un devoir d'humanité que remplissent les Alliés en allant, par delà les océans, secourir les hérö̈ques légions tchécoslovaques en lutte avec les germano-bolcheviks et tendre la main à leurs frères malheureux de Russie qui subissent les atroces persécutions bolchevistes [...]. En allant en Sibérie et en Russie, ils participent directement à la bataille unique qui se livre dans le monde." 42

40 Eine Analyse der Haltung von Le Temps, L'Echo de Paris und Le Figaro um die Jahreswende 1918/ 19 wie auch der Parlamentsdebatten vom 27. und 29. 12. 1918 findet sich bei Mayer, Peacemaking, S. 303-308.

41 Zum Konsens ausführlich: Serge Berstein/Jean-Jacques Becker, Histoire de l'anticommunisme en France. Band 1: 1917-1940, Paris 1987; dies., L'anticommunisme en France, in: Vingtième Siècle 15 (1987), S. 17-27; Jean-Jacques Becker, La France en guerre (1914-1918). La grande mutation, Paris 1988, S. 101 f., 186-191; Ziebura, Léon Blum, S. 179 f., $186 \mathrm{ff}$.

42 Handschriftliche Ausarbeitung Reynauds: „La leçon de l'armistice bulgare. Agissons en Sibérie“, undatiert, auf Briefpapier des "Palace Hotel, San Francisco" zwischen dem 15. Januar und 10. Februar 1919 verfaßt (AN, 74 AP 6). Zum Begriff des „antibolschewistischen Kreuzzugs“: Marc Ferro, Der große Krieg. 1914-1918, Frankfurt 1988, S. 363-368, hier: 366. 